\title{
REVIEW
}

\section{High-field MRI of the Central Nervous System: Current Approaches to Clinical and Microscopic Imaging}

\author{
Makoto SASAKI $^{1 *}$, Takashi Inoue ${ }^{2}$, Koujiro Tohyama ${ }^{3}$, Hirobumi Oikawa ${ }^{1}$, \\ Shigeru EhARA ${ }^{1}$, and Akira OgawA ${ }^{2}$ \\ ${ }^{1}$ Department of Radiology, ${ }^{2}$ Department of Neurosurgery, ${ }^{3}$ Department of Neuroanatomy, \\ Iwate Medical University \\ 19-1 Uchimaru, Morioka, Iwate 020-8505, Japan
}

(Received September 16, 2003; Accepted September 25, 2003)

\begin{abstract}
Introduction of clinical high-field MRI has raised interest in the use of clinical imaging - the efficacy of which has not yet been fully established-in daily practice. A high signal-to-noise ratio and profound susceptibility effects can improve the spatial resolution and image contrast of clinical imaging, whereas the heating effects of the radio frequency tend to prolong acquisition time. As well, inhomogeneities in the static or local magnetic fields can have a negative effect on image quality. The $T_{1}$ prolongation may affect $T_{1}$ contrast yet improve the enhancement effect of gadolinium chelate and the inflow effect of MR angiography. High-contrast imaging, such as the short inversion-time inversion recovery technique, can provide excellent intra- and extracerebral contrast comparable to microscopic or macroscopic specimens. High-field systems can also be applied to microscopic imaging. High-field MRI is expected to have an increased clinical impact in the near future. Technological advances tailored to high-field systems, as well as the accumulation of scientific evidence, will be necessary to establish its predominance over conventional MRI.
\end{abstract}

\section{Keywords: high-field MRI, 3 tesla, central nervous system, short inversion-time inversion recovery, MR microscopy}

\section{Introduction}

High-field magnetic resonance imaging (MRI) is a recent advance in medical imaging. Following the U.S. Food and Drug Administration's approval of brain and whole-body imaging at 4 tesla or less, nearly 100 high-field scanners have been installed worldwide, and many have already been used in daily practice as well as in experimental situations. In Japan, interest in and expectations for the clinical impact of high-field MRI have been growing significantly since the Pharmaceutical Affairs Council of the Japanese Ministry of Health, Labor, and Welfare approved brain imaging at 3 tesla (3T) in February 2003.

The advantages of high-field MRI as a tool for neuroscientific research have already been established, especially in the area of functional imaging, which comprises functional MRI (fMRI). ${ }^{1,2}$

\footnotetext{
*Corresponding author, Phone: +81-19-651-5111, Fax: +8119-622-1091, E-mail: masasaki@iwate-med.ac.jp
}

However, its efficacy in clinical practice remains unclear, mainly because of the paucity of scientific evidence indicating its priority at 3 to 1.5 tesla (1.5T). ${ }^{3-7}$ In this review, we discuss the impact of high-field MRI on clinical imaging of the central nervous system (CNS) by describing the basic knowledge of high-field MRI and morphological imaging techniques at $3 \mathrm{~T}$ in daily practice. We also briefly introduce magnetic resonance (MR) microscopic imaging, a recent area of interest in high-field MRI.

\section{Influence of a Static Magnetic Field}

Developing a full understanding of the advantages as well as the disadvantages of high-field MRI requires knowledge of the influences of a static magnetic field $\left(\mathrm{B}_{0}\right)$ on MR images. ${ }^{8}$ It is well known that a high signal-to-noise ratio (SNR) is a major-and possibly the major-advantage of high-field MRI. The SNR is said to rise roughly in proportion to $B_{0}$ because the MR signal is propor- 
tionate to $\mathrm{B}_{0}$ squared (the combined effect of increasing excess protons and the Lamor frequency), whereas the noise is proportionate to $\mathrm{B}_{0}$. The high SNR at $3 \mathrm{~T}$, which is about twice that at $1.5 \mathrm{~T}$, provides images with a high spatial resolution and short acquisition time. The strong susceptibility effects at 3T can enhance the blood oxygen level dependent (BOLD) effects in $\mathrm{FMRI}^{1,2}$ and in BOLD MR venography; ${ }^{9}$ the negative enhancement effects of gadolinium chelate in perfusion MRI; and the low signal intensity of iron-containing lesions in routine images. On the other hand, image distortions and other susceptibility artifacts are pronounced, especially with echo planar imaging (EPI) techniques such as diffusion-weighted imaging (DWI) and fMRI. Parallel imaging, line scan and non-EPI techniques are necessary to minimize image deterioration in DWI and diffusion tensor imaging. An increase in the chemical shift in proportion to $\mathrm{B}_{0}$ can improve spectral resolution of MR spectroscopy at $3 \mathrm{~T},{ }^{7}$ whereas in comparison to $1.5 \mathrm{~T}$, spatial misregistration of fatty tissues is doubled and the echo time at in-phase between water and fat is halved. The heating effect of the radio frequency (RF) is increased at $B_{0}$ squared. The high specific absorption rate (SAR) at 3T, which is four times that at $1.5 \mathrm{~T}$, tends to place some limits on clinical imaging. To maintain the SAR under the limit for patient safety, it is usually necessary to compromise scanning parameters by reducing the number of slices, reducing the echo train length or lengthening the repetition time (TR). The $T_{1}$ relaxation time is said to be dependent on $B_{0}$. At $3 T$, the $T_{1}$ of brain tissues is generally 1.1 to 1.3 times longer than that at $1.5 \mathrm{~T} .{ }^{10}$ On the other hand, $T_{2}$ relaxation is independent of $B_{0}$, although it is somewhat related to $T_{1}$ relaxation. However, the apparent $T_{2}$ values of tissue and lesions, especially iron-containing nuclei, are shortened, mainly due to diffusion-mediated microscopic susceptibility effects. The nonuniformity of the RF magnetic field $\left(B_{1}\right)$ is also dependent on $B_{0}$, because the penetration of RF into the subjects is decreased at high frequency. ${ }^{11}$ At $3 \mathrm{~T}$, the $\mathrm{B}_{1}$ inhomogeneity and different flip angles between the surface and center of the subjects are not negligible, sometimes resulting in uneven distribution of the signal intensity. Other characteristics of the high magnetic field include strong motion artifacts, increased acoustic noise, the possibility of transient vestibular stimulation, and tremendous magnetic traction power.

\section{Conventional Imaging}

Fast spin-echo (FSE) techniques at 3T can easily provide high-resolution proton-density weighted images (PDWI), $\mathrm{T}_{2}$-weighted images $\left(\mathrm{T}_{2} \mathrm{WI}\right)$, and fluid-attenuated inversion recovery (FLAIR) with a matrix size of $512 \times 512$ and slice thickness of 3 to $4 \mathrm{~mm}$ (Figs. 1a, b, d). A relatively long TR and short effective echo time (TE) would be suitable at $3 T$ because $B_{1}$ heterogeneity effects are minimized and apparent $T_{2}$ shortening is compensated, respectively. Image contrast seems to be similar to that at $1.5 \mathrm{~T}$, but on $\mathrm{T}_{2} \mathrm{WI}$ and FLAIR the low signal intensity of iron-containing nuclei seems to be more prominent than at $1.5 \mathrm{~T}$ because of pronounced susceptibility effects. When FSE techniques are used, the SAR limitation may considerably restrict parameter settings concerning the number of slices, echo train length or TR, resulting in a prolonged acquisition time. Parallel imaging or novel techniques utilizing lower flip angles such as TRAPS (transition between pseudo steady states) are believed to be effective at avoiding the RF heating issue in FSE sequences. ${ }^{12}$

At $3 \mathrm{~T}$, high resolution $\mathrm{T}_{2} \mathrm{WI}$ with a conventional spin echo (SE) technique is also available with an acceptable acquisition time (Fig. 1c). The SAR of this technique is so small that a sufficient number of slices to cover the entire brain can be obtained with a slice thickness of 3 to $4 \mathrm{~mm}$. SE $\mathrm{T}_{2} \mathrm{WI}$ may be applicable as a routine imaging approach in daily practice at $3 \mathrm{~T}$. In addition, deep nuclei as well as white matter containing iron show remarkably low signal intensities as a result of apparent $T_{2}$ shortening due to diffusion-mediated susceptibility effects. ${ }^{13}$ The image contrast is comparable to that of iron-stained specimens (Figs. 2a, b). Iron-concentration weighted imaging at high spatial resolutions can be accomplished with the SE technique at 3T. $\mathrm{T}_{2}{ }^{*}$-weighted images obtained with gradient echo techniques are also promising at $3 \mathrm{~T}$. The detectability of microbleeding in arteriosclerosis or diffuse axonal injury is expected to be markedly improved. ${ }^{14}$

SE $T_{1}$-weighted images $\left(T_{1} W I\right)$ seem to be unsuitable for routine imaging at $3 \mathrm{~T}$ because of several shortcomings: the number of slices is too small to cover the entire brain due to the limitation of the SAR; the uneven signal distribution due to $B_{1}$ heterogeneity is prominent, especially at the center of the brain (Fig. 1e); and delay of magnetization recovery due to $T_{1}$ prolongation may cause deterioration of the SNR and reduce $\mathrm{T}_{1}$-dependent contrast under short TR conditions. As an alternative to $\mathrm{SE} \mathrm{T}_{1} \mathrm{WI}$, we usually use the $3 \mathrm{D} \mathrm{RF}$-spoiled gradient echo, which has a high SNR, lower SAR, less $B_{1}$ dependency, acceptable $T_{1}$ contrast, and multiplanar capability (Fig. 1f). The contrast 

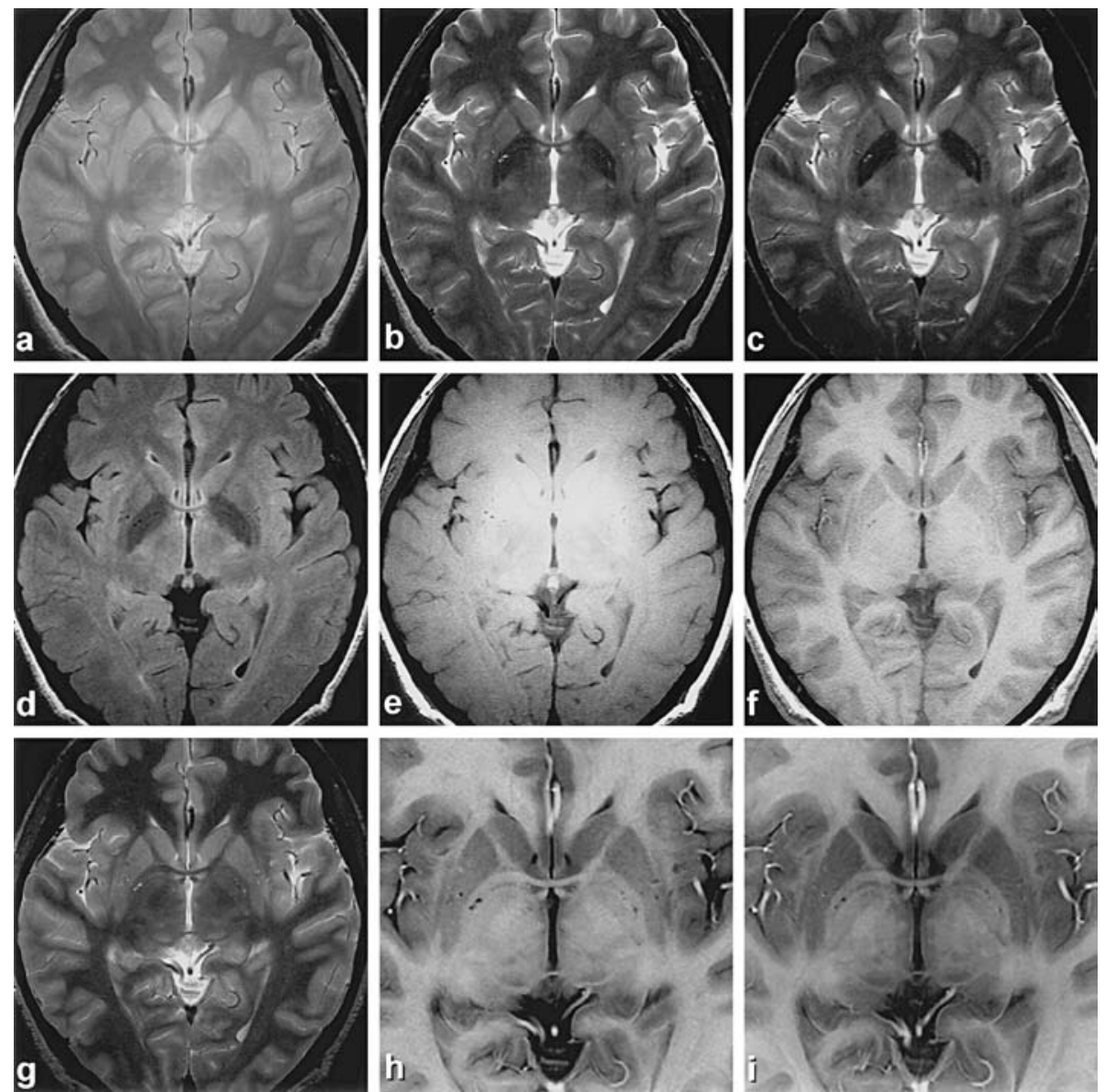

Fig. 1. Morphological brain images at 3T (healthy 31-year-old female)

Axial images of the same section with a slice thickness of $4 \mathrm{~mm}$, field of view of $22 \mathrm{~cm}$, and matrix size of $512 \times 256-384$.

a: FSE proton-density weighted image, b: FSE $\mathrm{T}_{2}$-weighted image, c: $\mathrm{SE} \mathrm{T}_{2}$ weighted image, d: FLAIR, e: $\mathrm{SE} \mathrm{T}_{1}$-weighted image, f: reformatted image from 3D RF-spoiled gradient echo, $\mathbf{g}$ : fast STIR, h: video-inverted fast STIR (the same image as $\mathbf{g}$, magnified), $\mathbf{I}: \mathrm{T}_{2}$-reversed (the same image as $\mathbf{a}$, videoinverted and magnified).

effects of gadolinium chelate may be enhanced at $3 \mathrm{~T}$, because the relaxivities of contrast agents $R_{1}$ and $R_{2}$ are largely constant in the range between $1.5 \mathrm{~T}$ and $3 \mathrm{~T} .^{3}$

The improved image quality of 3D time-of-flight MR angiography (MRA) at 3T is remarkable because of the high SNR and increased in-flow effects resulting from prolonged $T_{1}$ relaxation of the blood. ${ }^{4}$ Minute vessels such as anterior choroidal arteries and perforators are clearly visualized (Fig. 3). However, the SAR of magnetization transfer contrast (MTC) pulses is so high that TR should be significantly extended to reduce SAR under this limitation. Recently, novel techniques in which MT pulses apply only around the center of the k-space have succeeded in minimizing TR elongation by significantly reducing the SAR..$^{15,16}$

\section{High-contrast Imaging}

In daily practice, MRI examinations are performed with a combination of several kinds of images, including $\mathrm{T}_{1} \mathrm{WI}, \mathrm{T}_{2} \mathrm{WI}$, PDWI/FLAIR, DWI, and MRA. They provide complicated but useful data on lesion detection and characterization. However, the anatomical details of CNS structures in such images are sometimes lacking. It is therefore preferable that a new imaging technique be established that is capable of visualizing almost all the clinically required morphological information. To our knowledge, this was first proposed by Fujii and Nakada. ${ }^{17-19}$ They developed video-inverted high-resolution PDWI (4000/17), known as $T_{2}$-reversed imaging $\left(T_{2} R\right)$, as a routine $3 \mathrm{~T}$ imaging technique (Fig. 1i). $T_{2} R$ has superb contrast and spatial resolution mimicking gross 

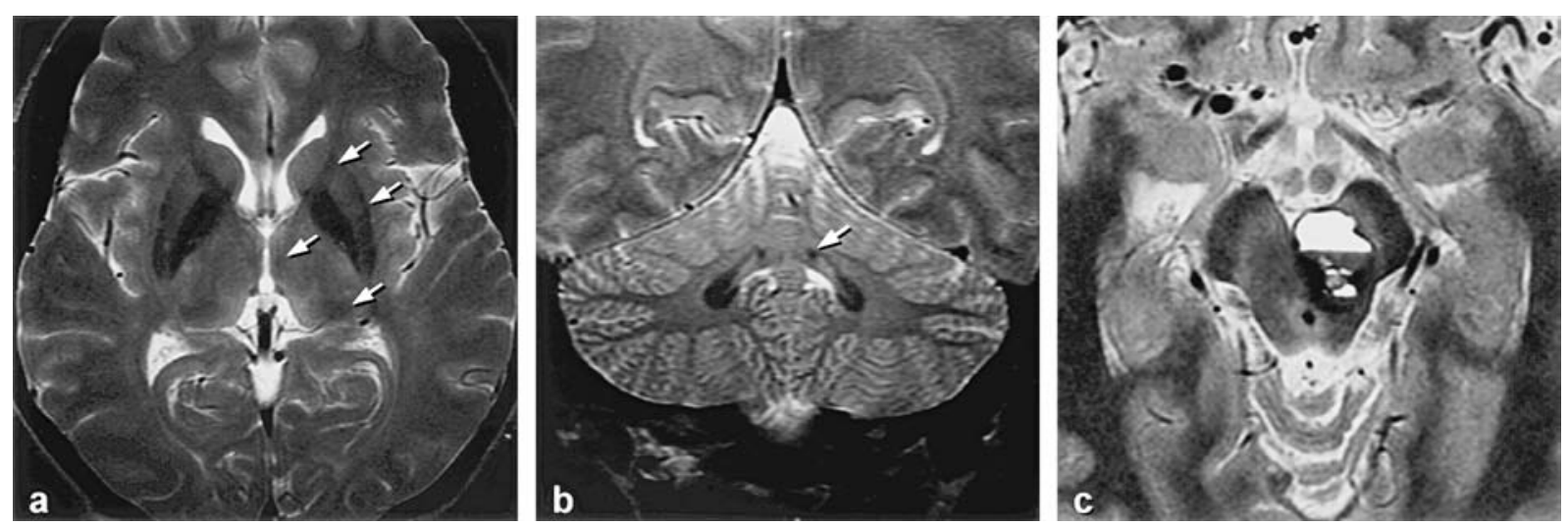

Fig. 2. Susceptibility effects in SE and FSE images at $3 T$

a: $\mathrm{SE} \mathrm{T}_{2}$-weighted image (healthy 31 -year-old female). The globus pallidus shows low signal intensity (arrows), as do the putamen, anterior limb of the internal capsule, dorsomedial nucleus, and pulvinar. b: Coronal SE $\mathrm{T}_{2}$-weighted image (the same volunteer as in a). The emboliform nucleus can be clearly identified (arrow) superomedial to the dentate nucleus.

c: Fast STIR image (ruptured cavernous hemangioma, 51-year-old female).

A low signal intensity suggesting a hemosiderin rim is evident. The fresh intratumoral hemorrhage shows the fluid-fluid level between the high and low signal intensity areas.
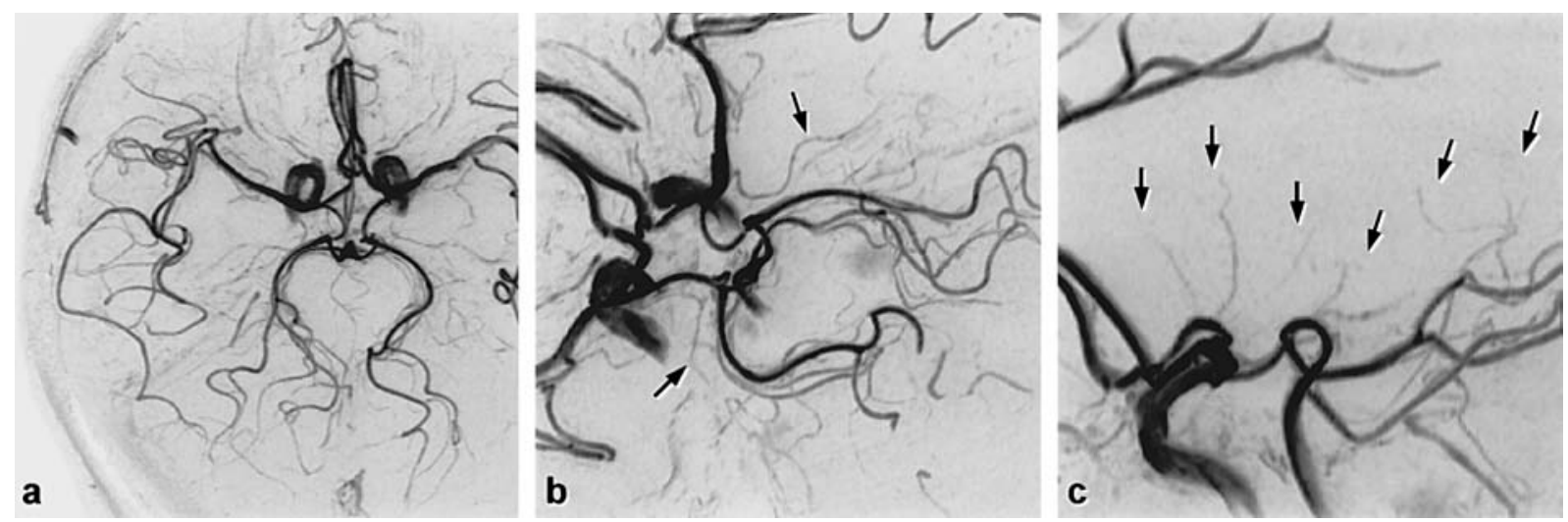

Fig. 3. 3D time-of-flight MR angiography with optimized magnetization transfer pulses at $3 \mathrm{~T}$ (healthy 39-year-old female)

Visualization of cortical branches is excellent (a). Anterior choroidal arteries (b: arrows) and perforating arteries (c: arrows) are also clearly depicted.

specimens, which makes it possible to visualize minute intracerebral and extracerebral structures because of the inherently high SNR and contrast of PDWI as well as the improved conspicuity provided by video-reversal processing.

As an alternative to $T_{2} R$, we proposed a fast short inversion-time inversion recovery (STIR) technique. ${ }^{20-22}$ The STIR sequence is widely known as a fat suppression technique, but it has high contrast resolution because the proton density and $T_{1}$ and $T_{2}$ relaxations have synergetic effects on image contrast. The SNR, which is considered relatively low, can be increased by utilizing FSE technique with a longer TR and shorter inversion time. $\mathrm{T}_{1}$ prolongation of brain tissues at $3 \mathrm{~T}$ also contributes to an improved SNR. Acquired images show excellent intracerebral contrast nearly identical to that of the myelin-stained specimens (Fig. 1g). We can identify minute intracerebral structures such as the subthalamic nucleus, intrathalamic structures, and intrahippocampal components, which are not well identified by MRI (Fig. 4). Under reversed video, the fast STIR technique can successfully mimic $T_{2} R$ (Fig. 1h). Visualization of cerebral vessels and other extracerebral structures is excellent. The contrast of pathological lesions is also superb, because $T_{1}$ and $\mathrm{T}_{2}$ relaxations additively affect signal alteration. Even hemorrhagic lesions can be identified thanks to strong susceptibility effects under 3T (Fig. 2c). 

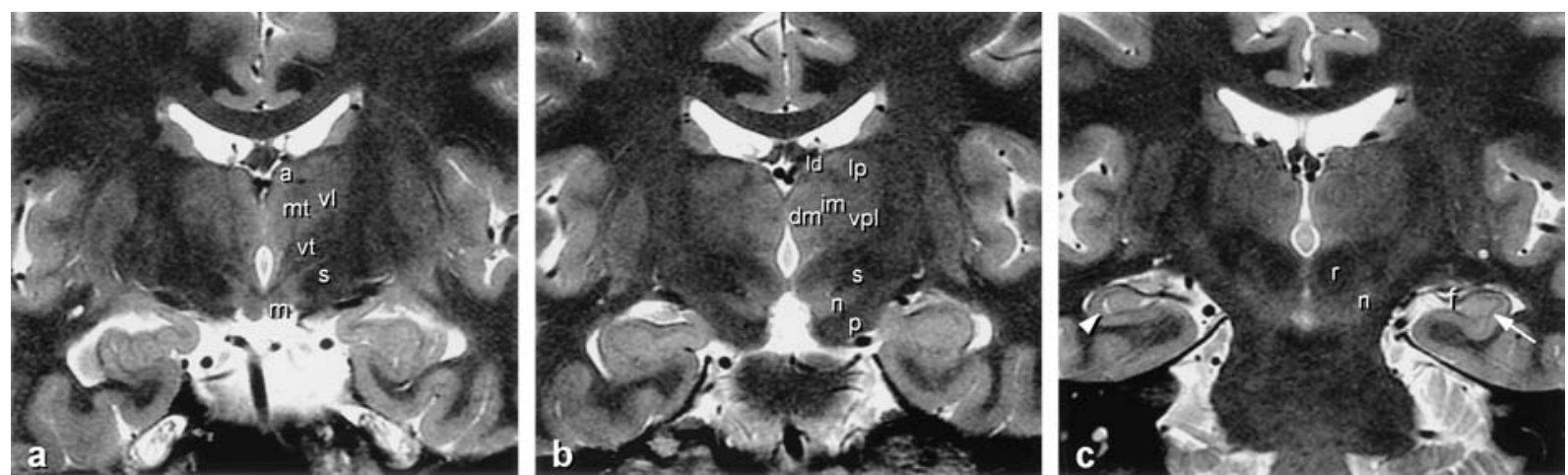

Fig. 4. High-resolution fast STIR images at 3T

a, b: Coronal contiguous images (healthy 54 -year-old female). The subthalamic nucleus (s) is clearly identified as a low signal intensity area below the ventral thalamus (vt). The circuit of Papez including the mamillary body (m), mamillothalamic tract $(\mathrm{mt})$, and anterior thalamic nucleus (a) is visualized. Other intrathalamic substructures, including the internal medullary lamina (im), dorsomedial nucleus (dm), ventrolateral nucleus (vl), lateral dorsal nucleus (ld), lateral posterior nucleus (lp), and ventral posterolateral nucleus (vpl), can also be seen. n: substantia nigra, p: cerebral peduncle.

c: Oblique coronal images perpendicular to the long hippocampal axis (healthy 57-year-old female). Intrahippocampal structures, such as the molecular layer of the cornu ammonis (arrow) and residual cavity of hippocampal sulcus (arrowhead), are well depicted. f: fimbria, n: substantia nigra, r: red nucleus.
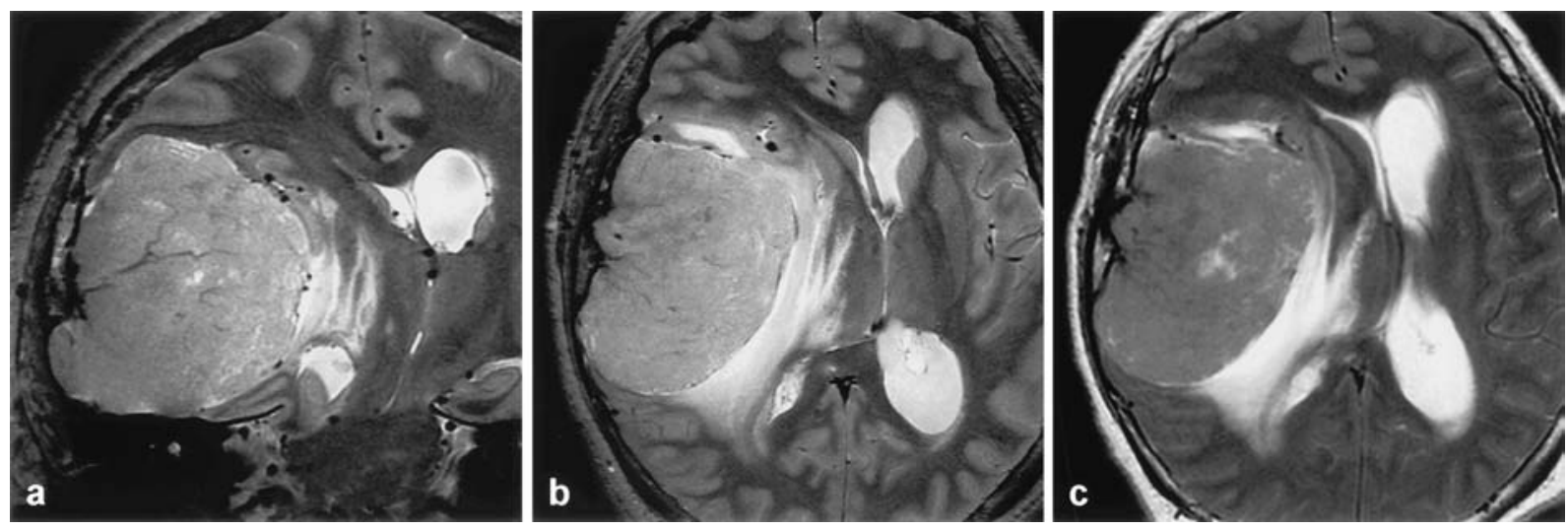

Fig. 5. Lower convexity meningioma (52-year-old female)

a, b: Fast STIR images at 3T, c: FSE $\mathrm{T}_{2}$-weighted image at $1.5 \mathrm{~T}$.

On fast STIR images at 3T, intratumoral textures, tumor vessels, and surrounding cerebral vessels and cerebral parenchyma are clearly visualized, revealing much more information than is available in images obtained at $1.5 \mathrm{~T}$.

As a preoperative means of assessing brain tumors, fast STIR can provide useful information concerning the tumor itself, tumor vessels, and surrounding vascular and parenchymal structures (Fig. 5). Fast STIR seems to be an all-round imaging technique at $3 \mathrm{~T}$ and can replace other conventional images in some situations of daily practice.

\section{Microscopic Imaging}

High-field MRI has the potential to achieve microscopic imaging. Recently, this field has marked several innovations. A perfusion technique using fixatives containing gadolinium chelate has allowed us to obtain high-resolution in-vitro volume images with a relatively short acquisition time. ${ }^{23,24}$ With this technique, the SNR of small animal brains can be dramatically increased by up to eight times. The image contrast depends mainly on the vasculature of tissues and resembles that of the above-mentioned STIR images (Fig. 6). Free unchelated manganese is utilized as a contrast agent or a neuroaxonal tracer ${ }^{25,26}$ In-vivo visualization of beta-amyloid plaque in transgenic mouse brains has been accomplished with iron-labeled peptides. ${ }^{27}$ 



Fig. 6. MR microscopic imaging at $3 T$

MR microscopic images of the rat $(\mathbf{a}, \mathbf{b})$ and cat (c) after perfusion by fixatives containing $5 \%$ and $2 \%$ Gd-DTPA. 3D $\mathrm{T}_{1}$-weighted FSE, voxel size: $0.3 \times 0.3 \times 0.3 \mathrm{~mm}$, acquisition time: 1.5 hours.

\section{Conclusions}

High-field MRI is a promising technique not only for scientific research, but also for clinical imaging of the CNS. Its clinical impact is expected to increase in the near future. However, its efficacy in diagnostic performance, patient outcome, and societal aspects has not yet been fully established. Optimization of imaging techniques and the accumulation of scientific evidence is clearly essential to future development.

\section{Acknowledgments}

This work was supported in part by Grants-inAid for Advanced Medical Science Research provided by the Ministry of Science, Education, Sports and Culture, Japan.

All images in this review were acquired with the approval of the Institutional Review Board. All volunteers and patients provided written consent before undergoing examination, and all animal experiments were performed according to protocols approved by the Animal Care and Use Committee.

\section{References}

1. Kim S, Ashe J, Georgopoulos AP, et al. Functional imaging of human motor cortex at high magnetic field. J Neurophysiol 1993; 69:297-302.

2. Turner R, Jezzard P, Wen H, et al. Functional mapping of the human visual cortex at 4 and 1.5 tesla using deoxygenation contrast EPI. Magn Reson Med 1993; 29:277-279.

3. Nobauer-Huhmann IM, Ba-Ssalamah A, Mlynarik $\mathrm{V}$, et al. Magnetic resonance imaging contrast enhancement of brain tumors at 3 tesla versus 1.5 tesla. Invest Radiol 2002; 37:114-119.

4. Bernstein MA, Huston III J, Lin C, Gibbs GF, Felmlee JP. High-resolution intracranial and cervical MRA at 3.0T: technical considerations and initial experience. Magn Reson Med 2001; 46:955-962.

5. Hunsche S, Moseley ME, Stoeter P, Hedehus M. Diffusion-tensor MR imaging at 1.5 and 3.0T: initial observations. Radiology 2001; 22:550-556.

6. Wang J, Alsop DC, Li L, et al. Comparison of quantitative perfusion imaging using arterial spin labeling at 1.5 and 4.0 Tesla. Magn Reson Med 2002; 48:242-254.

7. Gonen O, Gruber S, Li BSY, Mlynarik V, Moser E. Multivoxel 3D proton spectroscopy in the brain at 1.5 versus 3.0T: signal-to-noise ratio and resolution comparison. AJNR 2001; 22:1727-1731.

8. Ugurbil K, Garwood M, Ellermann J, et al. Imaging at high magnetic fields: initial experiences at 4T. Mang Reson Q 1993; 9: 259-277.

9. Barth M, Noebauer-Huhmann IM, Reichenbach JR, et al. High-resolution three-dimensional contrast-enhanced blood oxygenation level-dependent magnetic resonance venography of brain tumors at 3 Tesla: first clinical experience and comparison with 1.5 Tesla. Invest Radiol 2003; 38:409-414.

10. Wansapura JP, Holland SK, Dunn RS, Ball WS. NMR relaxation times in the human brain at 3.0 Tesla. J Magn Reson Imaging 1999; 9:531-538.

11. Alecci M, Collins CM, Smith MB, Jezzard P. Radio frequency magnetic field mapping of a 3 Tesla birdcage coil: experimental and theoretical dependence on sample properties. Magn Reson Med 2001; 46:379-385.

12. Hennig J, Weigel M, Scheffler K. Multiecho sequences with variable refocusing flip angles: optimization of signal behavior using smooth transitions between pseudo steady states (TRAPS). Magn Reson Med 2003; 49:527-535.

13. Schenck JF. Magnetic resonance imaging of brain iron. J Neurol Sci 2003; 207:99-102.

14. Scheid R, Preul C, Gruber O, Wiggins C, von Cramon DY. Diffuse axonal injury associated with chronic traumatic brain injury: evidence from $\mathrm{T}_{2}{ }^{*}$ weighted gradient-echo imaging at $3 \mathrm{~T}$. AJNR 
2003; 24:1049-1056.

15. Thomas SD, Al-Kwifi O, Emery DJ, Wilman AH. Application of magnetization transfer at 3.0T in three-demensional time-of-fligh magnetic resonance angiography of the intracranial arteries. J Magn Reson Imaging. 2002; 15:479-483.

16. Lin C, Bernstein MA, Gibbs GF, Huston III J. Reduction of RF power for magnetization transfer with optimized application of RF pulses in k-space. Magn Reson Med 2003; 50:114-121.

17. Fujii Y, Nakayama N, Nakada T. High-resolution $\mathrm{T}_{2}$-reverssed magnetic resonance imaging on high magnetic field system. J Neurosurg 1998; 89:492495.

18. Nakada T. High-field, high-resolution MR imaging of the human indusium griseum. AJNR 1999; 20: 524-525.

19. Harada A, Fujii Y, Yoneoka Y, Takeuchi S, Tanaka R, Nakada T. High-field magnetic resonance imaging in patients with moyamoya disease. J Neurosurg 2001; 94:233-237.

20. Sasaki M, Oikawa H, Ehara S, Tamakawa Y. Ventral thalamus and subthalamic nucleus: MR findings using optimized fast short inversion-time inversion-recovery technique. Radiology 2000; 217(P):672 (abstract).

21. Sasaki M, Oikawa H, Ehara S, Tamakawa Y. Internal structures of cerebral gray matter at 3 Tesla: MR imaging using fast short inversion-time inversion-recovery technique. Radiology 2001; 221(P):672 (abstract).

22. Oikawa H, Sasaki M, Tamakawa Y, et al. The substantia nigra in Parkinson disease: proton density-weighted spin-echo and fast short inversiontine inversion-recovery MR findings. AJNR 2002; 23:1747-1756.

23. Johnson GA, Cofer GP, Gewalt SL, Hedlund LW. Morphologic phenotyping with MR microscopy: the visible mouse. Radiology 2002; 222:789-793.

24. Johnson GA, Cofer GP, Fubara B, et al. Magnetic resonance histology for morphologic phenotyping. J Magn Reson Imaging 2002; 16:423-429.

25. Watanabe $T$, Natt $O$, Boretius $S$, Frahm J, Michaelis T. In vivo 3D MRI staining of mouse brain after subcutaneous application of $\mathrm{MnCl}_{2}$. Magn Reson Med 2002; 48:852-859.

26. Watanabe T, Michaelis T, Frahm J. Mapping of retinal projections in the living rat using high-resolution 3D gradient-echo MRI with Mn-induced contrast. Magn Reson Med 2001; 46:424-429.

27. Pautler RG, Mongeau R, Jacobs RE. In vivo transsynaptic tract tracing from the murine striatum and amygdala utilizing manganese enhanced MRI. Magn Reson Med 2003; 50:33-39.

28. Wadghiri YZ, Sigurdsson EM, Sadowski M, et al. Detection of Alzheimer's amyloid in transgenic mice using magnetic resonance microimaging. Magn Reson Med 2003; 50:293-302. 